\title{
Antibiotic treatment of metritis in dairy cows-A meta-analysis
}

\author{
P. Haimerl, S. Arlt, S. Borchardt, and W. Heuwieser ${ }^{1}$ \\ Clinic for Animal Reproduction, Faculty of Veterinary Medicine, Freie Universität Berlin, Berlin 14163, Germany
}

\begin{abstract}
The objective of this meta-analysis was to assess the efficacy of the treatment of bovine metritis with common antibiotic and nonantibiotic treatment options. Acute puerperal metritis, a systemic and potentially painful illness with rectal temperature $>39.5^{\circ} \mathrm{C}$ and signs of toxemia due to an infection of the uterus, occurs within $21 \mathrm{~d}$ after parturition. Because of the infectious nature, antibiotics are considered beneficial for the treatment of acute puerperal metritis. Each use of an antimicrobial drug, however, is associated with selective pressure for eventual emergence of resistant bacteria. The 23 trials evaluated in the course of a previously conducted systematic review were the basis for meta-analytic investigations. Selected trials were screened regarding their eligibility for the following investigations: (1) comparison of different antibiotic treatments with respect to metritis prevalence at time of re-examination, (2) efficacy of ceftiofur treatment with respect to metritis prevalence at time of re-examination, (3) comparison of efficacy of antibiotic versus nonantibiotic drugs with respect to metritis prevalence at time of re-examination, and (4) equivalence assessment of treatment effects on reproductive performance measures. Where at least 3 trials had investigated the same outcome variable and met the inclusion criteria (inclusion of a control or reference group diagnosed with metritis; reporting means and standard deviation in case of continuous data), meta-analytic investigations were carried out. Due to a shortage of comparable studies, we could not conduct investigations (1) and (3). Ceftiofur treatment of 828 metritic cows was associated with a decrease in the prevalence of metritis following treatment in comparison to 804 untreated cows. In conclusion, meta-analytic investigations uncovered a need for more high-quality studies. Furthermore, a positive effect of the most commonly used antibiotic
\end{abstract}

Received August 5, 2016.

Accepted January 15, 2017

${ }^{1}$ Corresponding author: w.heuwieser@fu-berlin.de drug, ceftiofur, for the treatment of bovine metritis could be shown. A comparison with other antibiotic or nonantibiotic treatment options could not be made. Key words: meta-analysis, acute metritis, antibiotic treatment, dairy cow

\section{INTRODUCTION}

Acute puerperal metritis (APM), an systemic illness with fever (rectal temperature $>39.5^{\circ} \mathrm{C}$ ) and signs of toxemia, occurs within $21 \mathrm{~d}$ after parturition and is characterized by an enlarged uterus and by an often fetid watery red-brown fluid to viscous off-white purulent uterine discharge (Sheldon et al., 2006). Depending on the definition for metritis, incidence risks range from 20 to 40\% (Haimerl and Heuwieser, 2014). Due to severe detrimental effects on reproductive performance and economics, APM is one of the most important postpartum diseases in dairy cows (Azawi, 2008; Overton and Fetrow, 2008; McLaughlin et al., 2013). A negative effect of APM on reproductive performance has been shown, especially in cases where APM results in endometritis. Increased time to pregnancy and higher culling rates were reported by LeBlanc et al. (2002), and a meta-analysis by Fourichon et al. (2000) found a decreased relative risk of pregnancy by 150 DIM and reduced pregnancy rates. Moreover, resulting reproductive inefficiency, culling, treatment, milk discard, labor, and increased risk of residues in food products cause substantial costs (LeBlanc, 2008).

A colonization of bacteria, such as Escherichia coli or Trueperella pyogenes, in the postpartum uterus is common (Földi et al., 2006; Miller et al., 2007; Bicalho et al., 2010; Santos et al., 2011). Depending on various factors such as bacterial pathogenicity and the cow's immune status, such colonization might result in an infection (Machado et al., 2014). Due to the mostly bacterial etiology, antibiotics are commonly considered beneficial for the treatment of APM (Beagley et al., 2010; Machado et al., 2012).

Antibiotics commonly used for the treatment of puerperal metritis include penicillin, third-generation cephalosporins, or a combination of ampicillin with oxytetracycline or cloxacillin (Nak et al., 2011). An- 
tibiotic resistance, however, has been recognized as a challenge for public health (Machado et al., 2014). Consequently, and because each application of an antimicrobial drug implies a risk of contributing to the emergence of resistant bacteria (Fishman, 2006; Ozawa et al., 2012), concern is growing regarding the potential effect of extensive antibiotic use in food-producing animals (Machado et al., 2014). Prudent drug use is of tremendous importance, especially concerning antibiotic agents valued for treating serious or life-threatening infections in human medicine.

Ideally, every decision made by a practitioner should be based on the latest and soundest scientific findings available (Glasziou et al., 1998; Arlt, 2016). The amount of available literature, however, is huge, mostly unstructured, and hardly manageable (Buchanan and Wooldridge, 2011). Moreover, usually several treatment options exist for one disorder (Caldwell et al., 2005); hence, information on the comparative effectiveness of treatment alternatives is needed (Pechlivanoglou et al., 2015). To provide such scientific results with the greatest possible reliability, the results of randomized, controlled trials (RCT) or meta-analyses of RCT are necessary (Arlt and Heuwieser, 2005).

Meta-analyses are systematic summaries and statistical analyses of a large collection of results from individual studies (Glass, 1976). As pointed out by Askie and Offringa (2015), one of the main reasons for undertaking systematic reviews and meta-analyses is to minimize bias by collating all available evidence on a particular topic. By synthesizing information from the greatest possible number of trials, and hence increasing the amount of available data (and thus statistical power), meta-analyses reduce the effect of randomness.

In a previously published paper on antibiotic treatment of metritis in dairy cows (Haimerl and Heuwieser, 2014) 23 trials were analyzed by means of a systematic review. Overall, the quality of the investigated studies was good, as indicated by a predominantly high evidence level (i.e., $83 \%$ were randomized controlled studies). However, the conducted review also revealed suboptimal consideration of critical issues, such as prudent antibiotic drug use, self-cure rates, and bacterial resistance.

Within the 23 trials, 10 different antibiotic agents were applied, with ceftiofur being the most frequent $(\mathrm{n}=17)$. Although efficacy of the antibiotics applied was specified in almost every study $(22 / 23)$, outcome parameters differed considerably (i.e., 12 and 6 studies assessed the effect on clinical and reproductive parameters, respectively). Even though 7 studies observed clinical improvement, none found improved reproduc- tive performance after an application of ceftiofur in metritic cows compared with the reference group.

Therefore, the overall objective of our study was the meta-analytic investigation of the efficacy of common treatment options. Specifically we set out (1) to compare the effect of various antibiotic treatments on metritis prevalence at time of re-examination after treatment, (2) to investigate the effect of ceftiofur treatment on metritis prevalence at time of re-examination compared with an untreated control, (3) to compare the efficacy of antibiotic with nonantibiotic drugs regarding metritis prevalence at re-examination after treatment, and (4) to compare reproductive performance measures between treated and nontreated cows. As a working hypothesis, we presumed that only a limited number of studies would be comparable concerning study design and measured outcome variables and hence eligible for meta-analytic investigations. Furthermore, we hypothesized a good success rate for treatment with ceftiofur regarding clinical cure and reproductive performance. We did not expect a significant advantage of ceftiofur treatment over other common antibiotic and nonantibiotic treatment options.

\section{MATERIALS AND METHODS}

\section{Literature Search}

Relevant steps and results of literature retrieval are illustrated in Figure 1. For a previously published review (Haimerl and Heuwieser, 2014), a comprehensive and systematic literature search was conducted on May 23, 2014, utilizing the databases PubMed (http:// www.pubmed.gov) and CAB Abstracts (http://ovidsp. tx.ovid.com/) to identify literature focusing on the antibiotic therapy of puerperal metritis in the cow. The subject heading "bovine metritis AND antibiotics" was used to find all trials on this topic written in the English or German language. To assure timeliness of the subsequent meta-analytic investigation, we conducted another literature search on December 6, 2015, utilizing the same databases, search terms, and search operators as in the previous trial.

\section{Inclusion and Exclusion Criteria}

As reported previously (Haimerl and Heuwieser, 2014), specific exclusion criteria were defined to exclude articles that did not describe clinical trials (e.g., descriptive or in vitro studies). Also, conference proceedings and review articles were excluded. Furthermore, articles not meeting the inclusion criteria, 

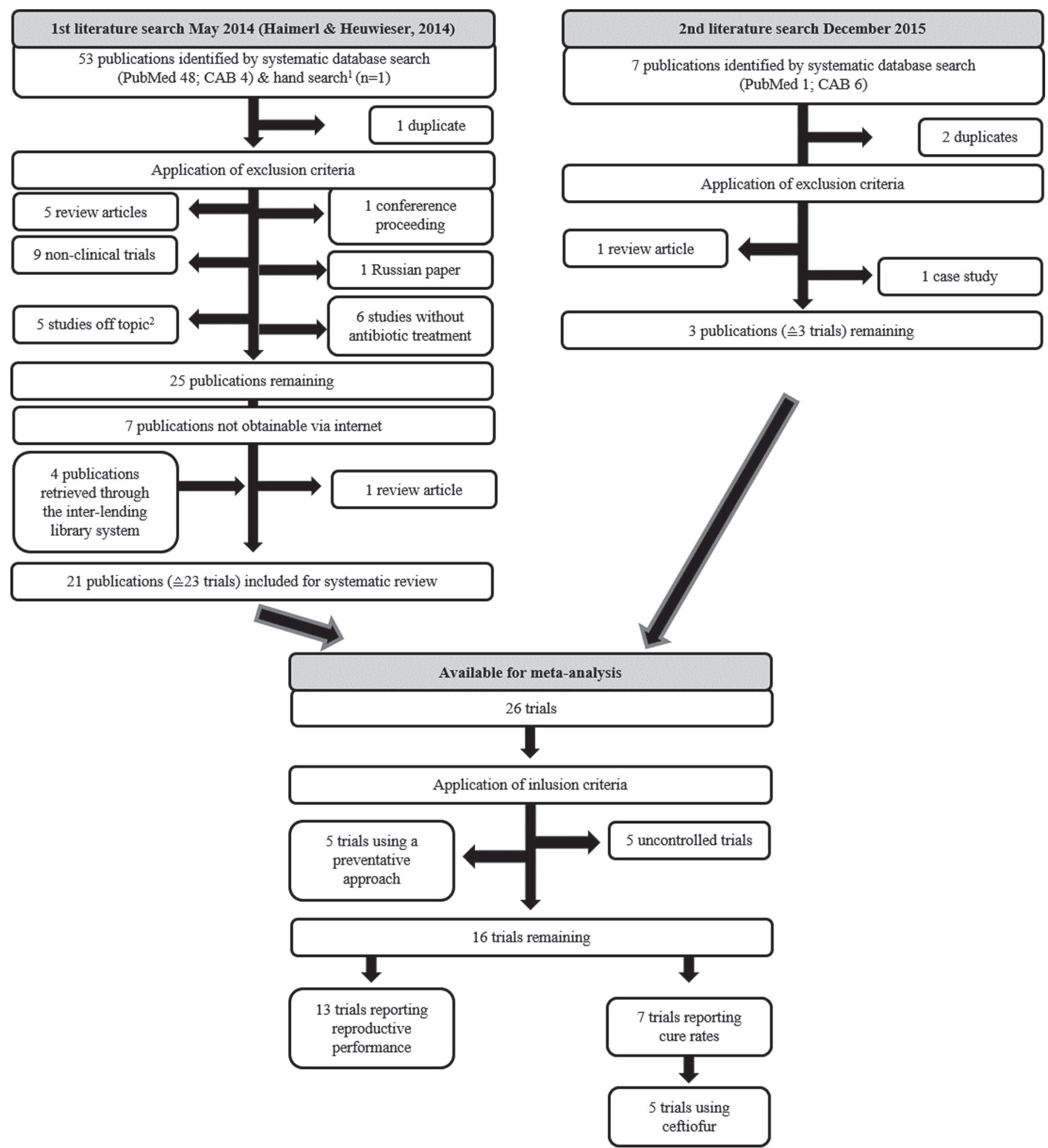

Figure 1. Search and selection criteria for literature using databases [PubMed (http://www.pubmed.gov) and CAB Abstracts (http://ovidsp. tx.ovid.com/)] on antibiotic treatment of acute puerperal metritis in dairy cows for meta-analytic investigation. Hand search $=$ search in the reference lists of selected articles; off-topic $=$ wrongly indexed articles 
due to wrong indexation ("off topic"), and those not obtainable through the internet, bibliographies, or interlending services were excluded as well. If multiple publications were retrieved describing the same trial, those containing the least information were regarded as doublets and excluded. Retrieval and management of references was performed with Endnote (Version X4 for Windows, Thomson Reuters, New York, NY).

Screening the 7 publications found in the course of the second database search revealed that 4 publications had to be rejected due to exclusion criteria (Figure 1). Specifically, the newly retrieved publications included 1 review found in both databases (Reppert, 2015), 1 case study (Chaudhari et al., 2012), and 1 publication already found in PubMed in May 2014 but referenced belatedly in CAB (Nak et al., 2011). The remaining 3 publications (containing 1 trial each) and the 23 trials selected previously resulted in 26 trials available for meta-analytic investigations.

Finally, inclusion criteria for being considered for the meta-analytic investigation were the presence of a control group, either untreated or treated by a reference treatment. In that regard, we considered no treatment and placebo treatment to be equivalent. Studies pursuing a preventative approach were excluded. After the application of inclusion criteria on the 26 selected trials, 10 trials were excluded due to being either uncontrolled ( $\mathrm{n}=5$; i.e., all cows received the same treatment) or implementing a preventative approach $(\mathrm{n}=5)$. All 3 trials available from the second literature search were uncontrolled (Freeman, 1955; Morcos et al., 1988; Yadav, 2014). Consequently, none of those 7 publications were eligible for meta-analytic investigations.

Of the remaining 16 trials, 7 reported a cure rate or a treatment effect on metritis prevalence (6 of these used ceftiofur as the treatment). Re-examinations were conducted within the first $7 \mathrm{~d}$ after the start of treatment (Drillich et al., 2001; McLaughlin et al., 2012; Sannmann et al., 2013; Lima et al., 2014), at 21 DIM (Giuliodori et al., 2013), or 4 (Steffan et al., 1984) or 7 wk after treatment (Jeremejeva et al., 2012). In addition, for 7 trials various reproductive performance parameters were reported. Reported cure rates were converted into metritis prevalence at examination after treatment to increase number of eligible trials and statistical power.

A predefined basic prerequisite for a given metaanalytic investigation was the presence of at least 3 trials investigating the same outcome variable, including a control or reference group diagnosed with metritis and reporting means and standard deviation in case of continuous data. In case of eligible trials with missing data necessary for meta-analytic investigations, authors were contacted.

\section{Data Extraction}

In the previous review (Haimerl and Heuwieser, 2014), trials remaining after the application of inclusion and exclusion criteria $(\mathrm{n}=23)$ were evaluated according to various evidence parameters and clinical aspects (Table 1).

\section{Statistical Analysis}

Frequency distributions regarding evidence and clinical aspects were compiled using SPSS for Windows (Version 22.0; SPSS Inc., Munich, Germany). For metaanalytic investigations, data for each trial meeting the inclusion criteria were extracted and analyzed using meta-analysis software Review Manager (Version 5.3, The Nordic Cochrane Centre, The Cochrane Collaboration, Copenhagen, Denmark).

Applying the effect size method (Hedges and Olkin, 1985), estimated effect sizes of chosen treatments were calculated for selected outcome measures. The following investigations were carried out: (1) comparison of different antibiotic treatments regarding metritis prevalence, (2) efficacy of a ceftiofur treatment regarding metritis prevalence, (3) comparison of efficacy of antibiotic versus nonantibiotic drugs regarding metritis prevalence, and (4) equivalence assessment of treatment effects on reproductive performance measures.

Odds ratios were calculated for treatment effects on metritis prevalence, whereas mean differences were calculated to estimate the treatment effect on reproductive performance. In addition to single study effects, an overall effect size, weighted by sample size, was calculated. Furthermore, a weighted mean difference and $95 \%$ confidence interval (CI) was calculated for each study outcome (Hedges and Olkin, 1985). Variation in experiment-level effect size was assessed with a $\chi^{2}$ test for heterogeneity (Duffield et al., 2008). Degree of heterogeneity of results among trials was quantified using the $\mathrm{I}^{2}$ statistic (Higgins et al., 2003). The $\mathrm{I}^{2}$ statistic describes the percentage of total variation across studies that is due to heterogeneity rather than chance. Where $\mathrm{Q}$ is the $\chi^{2}$ heterogeneity statistic and $\mathrm{k}$ is the number of trials, $\mathrm{I}^{2}$ was calculated as

$$
\mathrm{I}^{2}=\frac{\mathrm{Q}-(\mathrm{k}-1)}{\mathrm{Q}} \times 100 .
$$

Negative values of $\mathrm{I}^{2}$ were put equal to zero; consequently, $\mathrm{I}^{2}$ lay between 0 and $100 \%$. A value greater than $50 \%$ may be considered substantial heterogeneity (Duffield et al., 2008). 
Because included studies were expected to be heterogeneous, results were reported using the random effects model. A random effects meta-analysis assumes a normal distribution of the study effects (i.e., it allows that the true effect could vary from study to study; Borenstein et al., 2009). In cases of moderate or substantial heterogeneity, sources were explored using subgroup analysis, when trials were considered sufficiently homogeneous to be assessed together in a subgroup based on the study characteristics. Subgroup analysis was prespecified and conducted by considering evidence criteria, such as the inclusion in the study of a sample size calculation, randomization, and blinding of study groups, and relevant aspects of diagnostics, such as bacteriological examination (yes/no) and antimicrobial testing (yes/no). To investigate the possible effect of large weight and outlying results on the overall estimated effect obtained from the meta-analysis, where applicable, a sensitivity analysis was performed by eliminating the studies with the largest weights or outlying results one by one. Forest plots were used to visually display the estimated effect size (Z), 95\% CI, and study weights. The presence of publication bias was investigated graphically using funnel plots, in which

Table 1. Checklist used to evaluate publications on acute puerperal metritis in dairy cows considering the level of evidence

\begin{tabular}{|c|c|}
\hline Bibliography & Possible input parameters \\
\hline \multicolumn{2}{|l|}{ Paper identification } \\
\hline \multicolumn{2}{|l|}{ Author } \\
\hline \multicolumn{2}{|l|}{ Title } \\
\hline \multicolumn{2}{|l|}{ Journal } \\
\hline \multicolumn{2}{|l|}{ Year of publication } \\
\hline \multicolumn{2}{|l|}{ Materials and methods } \\
\hline Sample size & Number \\
\hline Stage of evidence & 1 to $4^{1}$ \\
\hline Blinding & Yes/No \\
\hline Randomization & Yes/No \\
\hline Control group & Yes/No \\
\hline Use of antibiotic & Preventive/therapeutic/other ${ }^{2}$ \\
\hline Size of antibiotic treatment group & Number \\
\hline Size of reference group & Number \\
\hline Size of untreated control group & Number \\
\hline \multicolumn{2}{|l|}{ Antibiotic agent } \\
\hline \multicolumn{2}{|l|}{ Dosage } \\
\hline Start and duration of treatment & $\mathrm{DIM} /$ days \\
\hline Type of treatment & Systemic/local \\
\hline Bacteriological examination & Yes/No \\
\hline Minimum inhibitory concentration & Yes/No \\
\hline Susceptibility test & Yes/No \\
\hline Disk diffusion method & Yes/No \\
\hline Microdilution method & Yes/No \\
\hline \multicolumn{2}{|l|}{ Reference treatment } \\
\hline \multicolumn{2}{|l|}{ Dosage } \\
\hline Start and duration of treatment & DIM/days \\
\hline \multicolumn{2}{|l|}{ Efficacy of antibiotic treatment } \\
\hline \multicolumn{2}{|l|}{ Efficacy of reference treatment } \\
\hline \multicolumn{2}{|l|}{ Outcome variable(s) } \\
\hline Self-cure rate & $\%$ \\
\hline \multicolumn{2}{|l|}{ Topics discussed } \\
\hline Applied antibiotic as "antibiotic of last resort" & Author's statement/No \\
\hline Antibiotic efficacy & Author's statement/No \\
\hline Bacterial load & Author's statement/No \\
\hline Bacteriological examination & Author's statement/No \\
\hline Minimum inhibitory concentration & Author's statement/No \\
\hline Bacterial resistence & Author's statement/No \\
\hline Prudent use of antibiotics & Author's statement/No \\
\hline Prudent drug use guidelines & Author's statement/No \\
\hline Alternative treatments/reference treatments & Author's statement/No \\
\hline Self-cure & Author's statement/No \\
\hline Is there an indication of financial support? & Name of company/No \\
\hline
\end{tabular}

${ }^{1}$ Levels of research evidence according to Bassler and Antes, 2000. 1 = Meta-analyses of randomized, controlled trials (Ia); randomized, controlled trials (Ib). 2 = Controlled trials without randomization (IIa); well-designed, quasi-experimental studies (IIb). 3 = Well-designed, nonexperimental descriptive studies (e.g., case-control studies). 4 = Opinions of respected authorities, reports of expert committees, clinical experience.

${ }^{2}$ Studies describing other aspects such as pharmacokinetics, pharmacodynamics, pathogen load. 


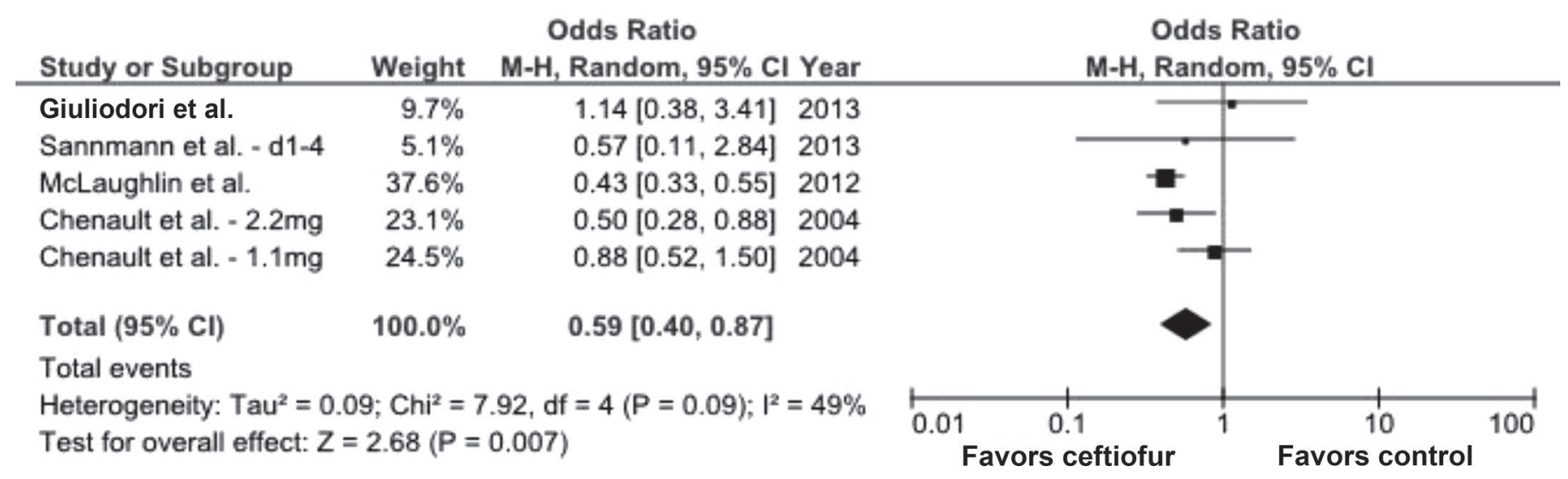

Figure 2. Forest plot on the effect of ceftiofur treatment on the prevalence of bovine metritis $(\mathrm{n}=1,632)$ at the time of re-examination. Odds ratios were calculated by means of the Mantel-Haenszel method (M-H). Each square represents the mean effect size for a study. The upper and lower limit of the line connected to the square represents the upper and lower 95\% CI for the effect size. The size of the square reflects the relative weighting of the study to the overall effect size estimate, with larger squares representing greater weight. The diamond at the bottom represents the overall effect size (location of the diamond) and its $95 \%$ CI (width of the diamond). The solid vertical line represents an odds ratio of zero or no effect. Squares located on the left side of this line represent studies showing an effect in the group treated with the former treatment, whereas squares located on the right side of this line indicate an effect found in the reference group. Study or subgroup refers to the treatments compared. Study or subgroup refers to the first author of the publication. The 2 experiments published by Chenault et al. (2004) originate from one publication and only differ in the dose applied. In the study conducted by Sannmann et al. (2013), only those cows treated between $\mathrm{d} 1$ and 4 postpartum could be included into the meta-analysis.

the size of effect for each treatment and control group comparison was plotted against its standard error and the resulting plot observed for deficiencies in predicted funnel shape.

\section{Comparison of Antibiotic Treatments}

Only 2 of the preselected trials compared the effect of various antibiotic treatments on metritis prevalence within the first days after treatment. Hence, investigating this approach a by means of meta-analysis was not feasible.

\section{Comparison of Efficacy of Antibiotic Versus Nonantibiotic Drugs}

Screening of the retrieved publications considering inclusion and exclusion criteria provided 2 trials comparing metritis prevalence after an antibiotic versus an alternative treatment. Hence, a meta-analytic investigation of this objective was not feasible.

\section{RESULTS}

\section{Efficacy of a Ceftiofur Treatment}

Due to incomplete reporting (Drillich et al., 2007) and the absence of a control group diagnosed with metritis (Drillich et al., 2001, 2007), only 4 of the 6 publications identified remained for analysis. Because 1 publication entailed 2 experiments (Chenault et al., 2004), a total of 5 trials including 1,632 metritic cows were eligible for the investigation of the effect of a ceftiofur treatment on metritis prevalence.

Analysis of the included trials revealed a significantly decreased prevalence of metritis after a ceftiofur treatment $(\mathrm{n}=828)$ in comparison to an untreated control group $(\mathrm{n}=804 ; \mathrm{Z}=2.68,95 \% \mathrm{CI}=0.40$ to $0.87 ; P=$ 0.007; Figure 2). The width of the $95 \%$ CI (based on SD) varied considerably between studies. For 3 trials (Chenault et al., 2004; Giuliodori et al., 2013; Sannmann et al., 2013) CI including zero could be found. Heterogeneity was present $\left(\chi^{2}=7.92, \mathrm{df}=4, P<0.09\right.$, $\mathrm{I}^{2}=49 \%$ ); therefore, sources of heterogeneity were explored with a subgroup analysis.

Subgroup Analysis. Because all of the 5 analyzed studies were randomized and none was fully blinded, a formation of subgroups based on those criteria was not possible. Analysis of subgroups considering an a priori sample size calculation did not reveal a source of heterogeneity (Figure 3). In contrast, formation of subgroups based on sample size determination resulted in an increase in heterogeneity in the group of trials having conducted a sample size calculation. A reduction in heterogeneity occurred when trials with a sample size calculation were removed. Also, considering relevant diagnostic aspects, such as bacteriological examination or susceptibility testing, subgroups could not be formed because only in 1 (Sannmann et al., 2013) of the 5 trials was that examination performed.

Sensitivity Analysis. Sensitivity analysis, performed by excluding the trial with the highest weight, 
and hence having the strongest statistical effect (McLaughlin et al., 2012), did improve the homogeneity considerably $\left(\mathrm{I}^{2}=0 \%\right)$. The initially significant effect $(\mathrm{Z}=2.68 ; P=0.007$ vs. $\mathrm{Z}=1.91 ; P=0.06)$ and the variance $\left(\tau^{2}=0.09\right.$ vs. 0.00$)$ were also reduced. When excluding the trial showing most outlying results (Giuliodori et al., 2013), effect size could be increased to $\mathrm{Z}=3.13(P=0.002)$, whereas variance was reduced $\left(\tau^{2}=0.07\right)$.

Publication Bias. Concerning the effect of a ceftiofur treatment on metritis prevalence, the presented funnel plot (Figure 4) revealed an even distribution of the studies around the mean effect size. This suggests that a publication bias toward studies with higher standard error (i.e., usually smaller studies; seen as a funnel shape) is not apparent.

\section{Investigations Regarding Reproductive Performance Measures}

Screening of the retrieved publications considering inclusion and exclusion criteria did not yield more than 2 trials assessing the effect of an antibiotic or alternative treatment or both on one respective reproductive performance measure, except for days open. Three studies investigated a calving to conception interval after the treatment of metritic cows. Because 1 of those studies (Goshen and Shpigel, 2006) did not report a standard deviation, which is necessary for statistical analysis, we contacted the authors. Unfortunately, our attempts to obtain missing data were not successful. Hence, 2 studies yielding 2 comparisons and including 107 metritic cows were eligible for statistical evaluation. Overall, 3 of the 4 investigated treatment options revealed a treatment effect concerning the calving to conception interval (Figure 5). Statistical significance $(P=0.006)$ could be shown for the combination therapy of ceftiofur and $\mathrm{PGF}_{2 \alpha}$ (Jeremejeva et al., 2012). Conversely, the same publication reported a negative effect in a reference group treated with ceftiofur and flunixin.

\section{DISCUSSION}

Most recently APM has been associated with visceral pain (Stojkov et al., 2015), underscoring the importance of the disease as an animal welfare issue and

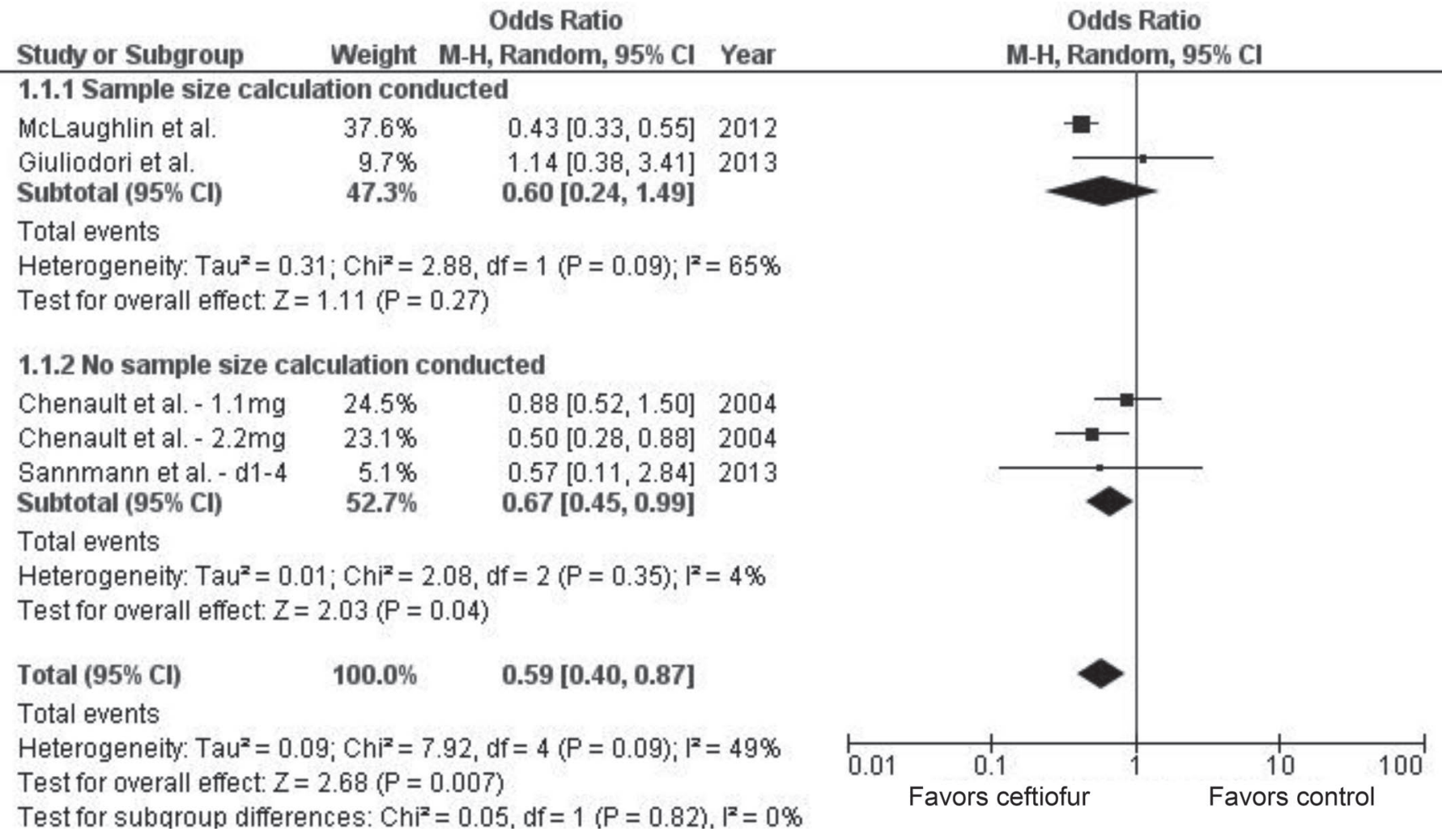

Figure 3. Forest plot of the effect of ceftiofur on the prevalence of bovine metritis at the time of re-examination. Odds ratios were calculated by means of the Mantel-Haenszel method (M-H). Subgroups were created according sample size estimation (yes/no). The two upper diamonds represent the overall effect sizes within the respective subgroups, whereas the diamond at the bottom represents the overall estimate of all studies. Boxes and whiskers as defined in Figure 2. 

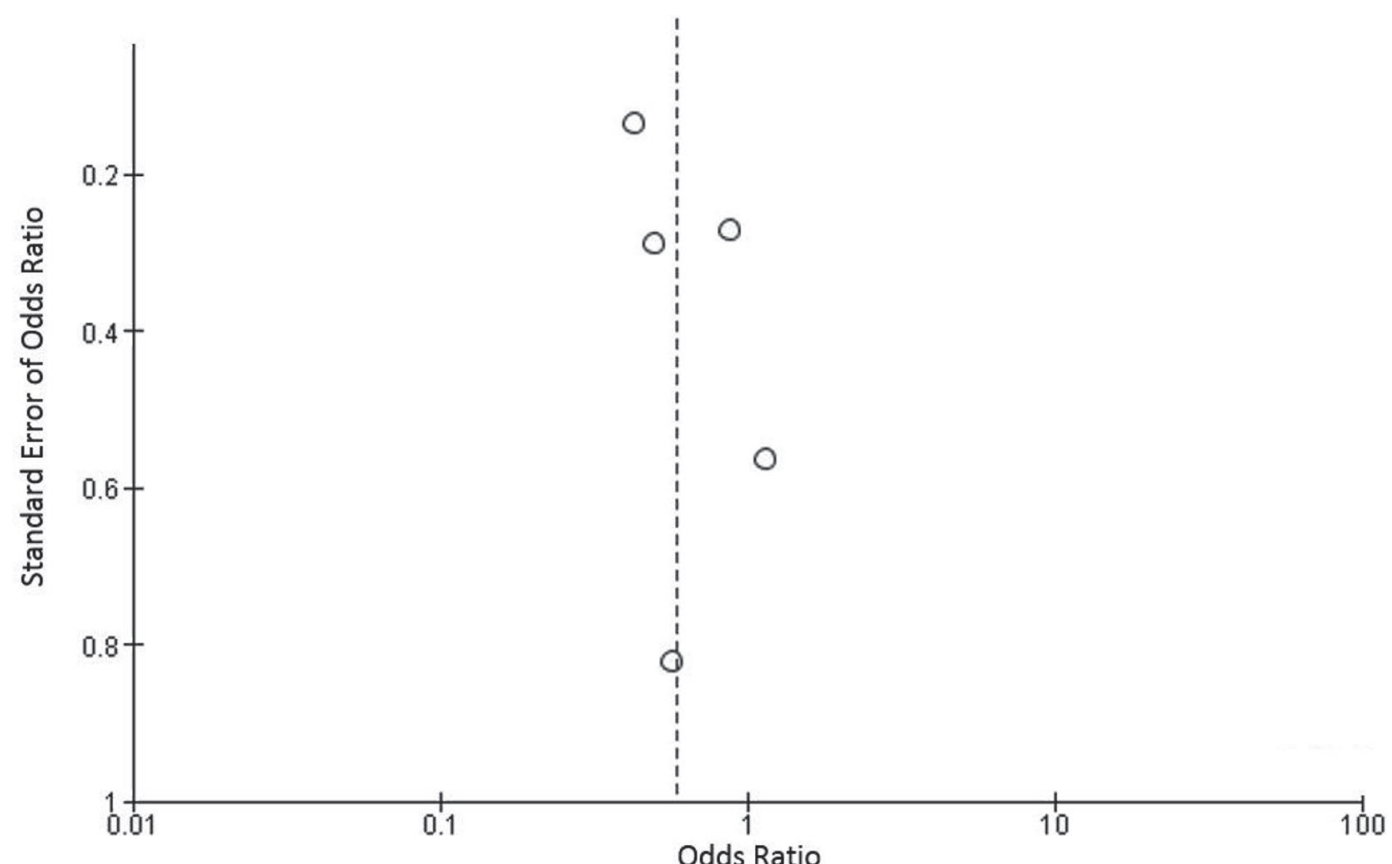

Figure 4. Funnel plot of ceftiofur effect on metritis prevalence at the time of re-examination for assessing publication bias. The vertical line represents the mean effect size. Publication bias may be present if there are an unequal number of studies (particularly smaller weight studies) on one side of the horizontal line.

consequently the necessity of an efficacious therapy. Its frequent treatment with antibiotics (Machado et al., 2012), however, is potentially contradictory to the recent demand for prudent antibiotic drug use. Hence, the present study was conducted to investigate and compare the efficacy of various treatment options by meta-analytic means.

As a working hypothesis, we presumed that only a low number of studies was comparable concerning study design and measured outcome variables and, hence, eligible for meta-analytic investigations. Furthermore, we hypothesized a good success rate after ceftiofur treatment regarding clinical cure and reproductive performance. Nevertheless, we did not expect a significant advantage of ceftiofur treatment over other common antibiotic and nonantibiotic treatment options.

In line with our first hypothesis, we did face a shortage of suitable trials. The number of studies eligible for meta-analytic investigation was very limited and did not exceed 5 trials for any of the chosen investigations. For some of the planned investigations the retrieved number of publications was less than 3 and, according to our prerequisites, did not allow meta-analytic investigation (i.e., comparison of antibiotic treatments

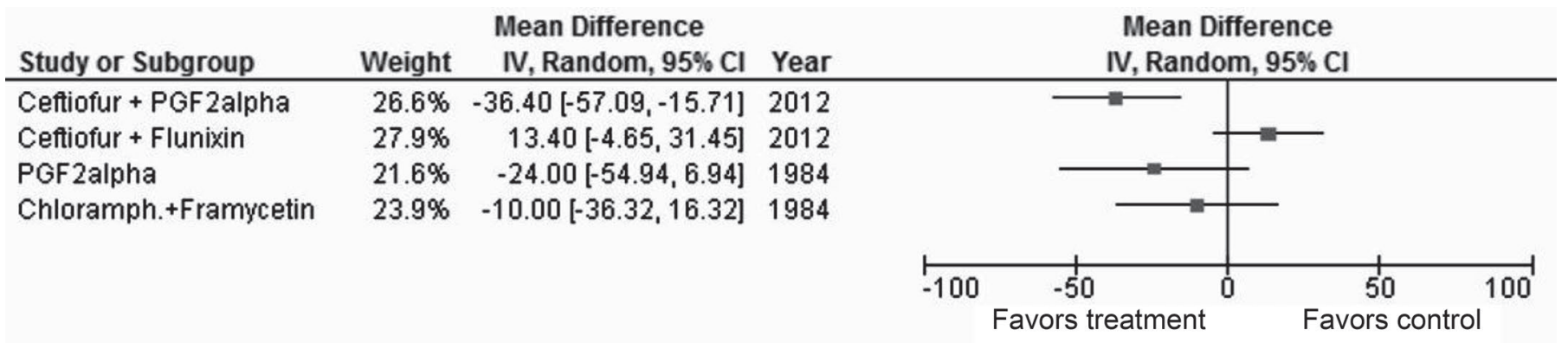

Figure 5. Forest plot of the effect of various treatment options on days open in dairy cows suffering from metritis (n $=107)$. The weight given to each study, represented by the size of the squares, is the inverse of the variance of the effect estimate (IV). The former 2 comparisons were derived from Jeremejeva et al. (2012), whereas the other 2 originate from Steffan et al. (1984). Boxes and whiskers as described for Figure 2. Chloramph. $=$ chloramphenicol. 
regarding metritis prevalence, direct comparison of efficacy of antibiotic versus nonantibiotic drugs regarding metritis prevalence and reproductive performance, and comparison of efficacy of single antibiotic treatments regarding reproductive performance). As reported by Lam and Kennedy (2005), meta-analytic investigations require a minimum of 2 eligible trials. As the validity of such analyses is highly questionable, we decided to require a minimum of 3 trials, being aware that even then evidence is suboptimal. Also, considering relevant diagnostic aspects, such as bacteriological examination or susceptibility testing, did not allow the formation of subgroups because only in 1 (Sannmann et al., 2013) of the 5 trials those examinations were performed. This observation further supported the shortage of well-designed comparable trials, it also points out suboptimal consideration of critical issues (Haimerl and Heuwieser, 2014) or incomprehensive reporting (Lean et al., 2016).

With regard to homogeneity, it is important to discuss the fact that in the different trials, different times for re-examination and different methods of determination of treatment success were used (Table 2). As stated earlier, times of re-examinations varied from $1 \mathrm{~d}$ up to $7 \mathrm{wk}$ after treatment. Hence, comparability of the results may be questioned. The objective, however, of any treatment of APM is a short-term but long-lasting success. Hence, an inclusion of prevalence measures based on different times relative to diagnosis are reasonable in this specific condition.

The fact that, of the 7 trials applying ceftiofur, different preparations were used is also open to criticism; preparations included ceftiofur hydrochloride (Chenault et al., 2004; Jeremejeva et al., 2012; Giuliodori et al., 2013) and ceftiofur crystalline-free acid (McLaughlin et al., 2012; Sannmann et al., 2013). Moreover, due to differing pharmaceutical regulations in various countries, dosages applied and duration of treatment varied among the studies applying ceftiofur hydrochloride. As all drug applications were subject to drug approval processes of the respective countries, we assumed that the treatment regimens used were supported by evidence of efficacy.

Definition and diagnosis of APM were highly homogeneous across the included studies and accorded with the definition proposed by Sheldon et al. (2009; i.e., including fever $>39.5^{\circ} \mathrm{C}$, signs of toxemia, an enlarged uterus and fetid uterine discharge within the first 21 $\mathrm{d}$ after parturition). One exception, however, is the trial conducted by Steffan et al. (1984). Those authors investigated cows that, by today's standards, would be classified as having purulent vaginal discharge or endometritis. Diagnostic procedures for the re-examinations were also fairly consistent (Table 2). In most studies, treatment success was defined as no escape therapy necessary, rectal temperature below $39.5^{\circ} \mathrm{C}$, and no vaginal discharge. In some studies additional parameters were evaluated. The lack of validated, consistent definitions and outcome variables stated by de Boer et al. (2014) regarding endometritis could not be confirmed for the scenario in our study.

One objective of the meta-analysis was the investigation of clinical cure and reproductive performance after a ceftiofur treatment. Confirming our second hypothesis, an overall significant decline in metritis prevalence subsequent to treatment could be detected. Confidence intervals including zero in 3 of the 5 trials suggested, however, the absence of a treatment effect (Figure 2). Two of those 3 studies had been assigned the lowest weights and thus did not affect overall effect size much; therefore, the treatment effect remained significant. Weights were assigned to each trial's result according to the precision of its treatment effect estimate. Trials having more precise estimates were more heavily weighted. In most cases, as in the present study, the precision of the estimates is primarily determined by the number of animals included. Trials including the largest sample sizes are, however, not necessarily those with the highest quality (Haimerl et al., 2013); therefore, the distribution of weight should be considered carefully. To address the possible effect of large weight on the overall estimated effect, a sensitivity analysis was performed by stepwise excluding the studies in a descending order of weight. Exclusion of the trial attributing the highest weight (McLaughlin et al., 2012) reduced a previously statistically significant effect of ceftiofur to a nonsignificant effect. This finding, however, was not surprising, as this trial showed the strongest positive treatment effect. Far more interesting, concerning robustness of the treatment effect, was the exclusion of the outlier. Its exclusion, resulting in a moderately increased treatment effect and slightly reduced variance, showed that the overall outcome was not biased by this outlier.

Investigations of clinical cure after ceftiofur treatment revealed moderate heterogeneity among the included studies $\left(\mathrm{I}^{2}=49 \%\right)$. To identify sources of heterogeneity, where feasible, subgroup analysis was performed. Formation of subgroups based on the mode of sample size determination surprisingly resulted in an increase in heterogeneity in the group of trials having conducted a sample size calculation. Although a quality criterion (Lean et al., 2016), a reduction in heterogeneity occurred when trials with a sample size calculation were removed. Those effects clearly show that a lacking sample size calculation was not responsible for detected heterogeneity. 


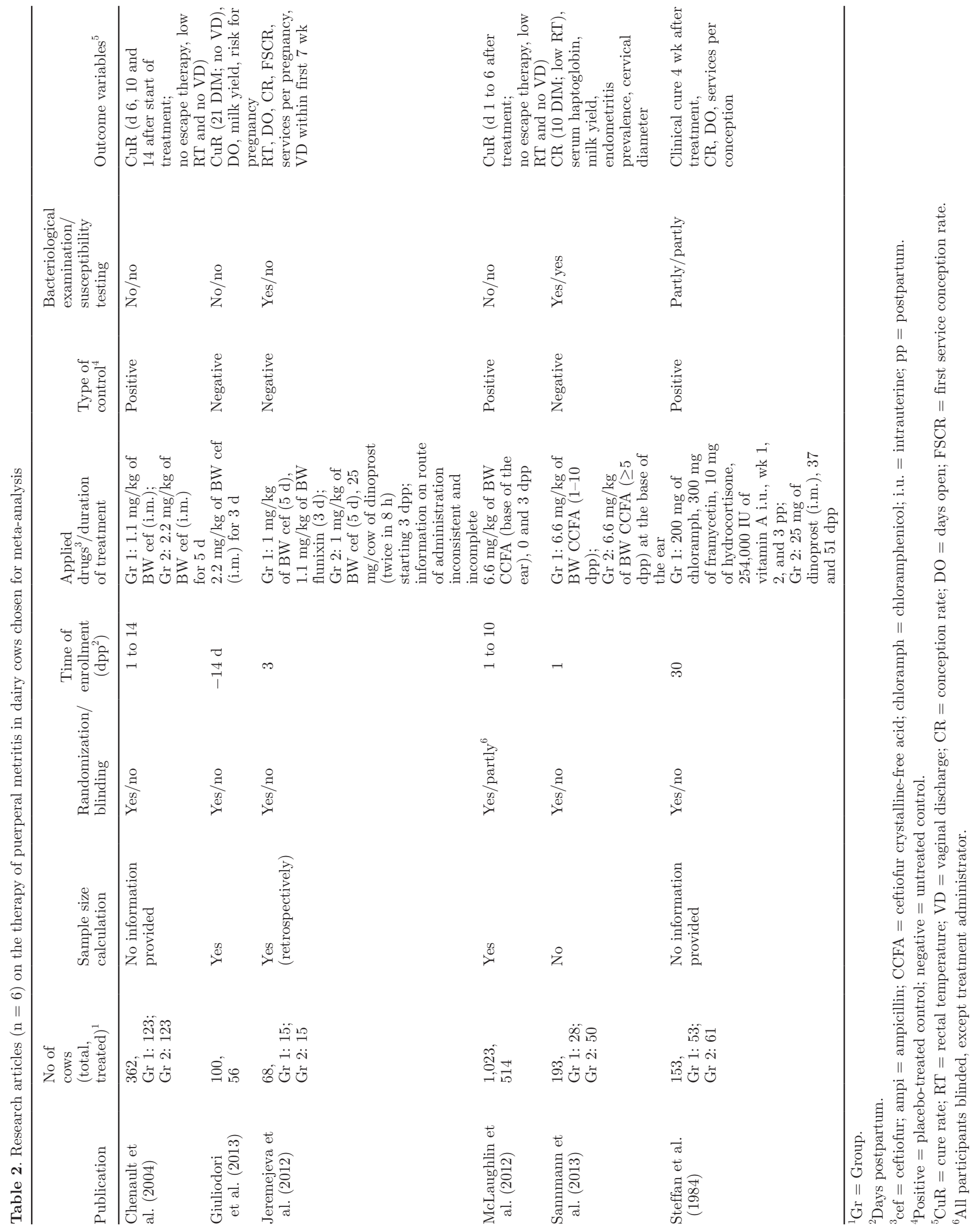


Due to a lack of suitable trials, we could not sufficiently compare the treatment effect of ceftiofur with non-antibiotic treatments; only a $\mathrm{PGF}_{2 \alpha}$ treatment might be advantageous over an antibiotic treatment, as illustrated in Figure 5. The number of antibiotic agents used for comparison, however, was limited (n $=3)$. In addition, a combined treatment with $\mathrm{PGF}_{2 \alpha}$ and ceftiofur showed an even stronger treatment effect than $\mathrm{PGF}_{2 \alpha}$ alone. One might deduce that this finding supports a concurrent treatment, including an antibiotic agent, rather than promoting a replacement of antibiotic drugs. As the comparisons, however, are from 2 different studies conducted 28 yr apart, and thus under different conditions, such an extrapolation has to be considered carefully. Furthermore, only combinations of different antibiotic agents were eligible for comparison. Thus, the efficacy of a single agent could not be assessed and might have been masked by the other agents.

A direct comparison between different antibiotic drugs was not possible due to a lack of suitable trials. Hence, it was not possible to meta-analytically determine if antibiotics could be used for the treatment of metritis that are being perceived as less critical than ceftiofur. A guideline by the World Health Organization classifies common antibiotic agents into critically important, highly important, and important antimicrobials (WHO, 2011). Concerning the critically important agents, fluoroquinolones and third- and fourth-generation cephalosporins, such as ceftiofur, data from the national monitoring initiative of the German Federal Office of Consumer Protection and Food Safety (BVL) reveal growing resistance in various bacteria (Federal Office of Consumer Protection and Food Safety, 2014). A general threat of emerging antimicrobial resistance of zoonotic organisms in food animals worldwide has been pointed out by several authors (Tragesser et al., 2006; Mann et al., 2011; Ozawa et al., 2012). In the Netherlands, the same genes encoding for extended spectrum beta-lactamases in E. coli isolates were found in food animals (especially poultry) and in humans with serious infections (WHO, 2011). Besides the awareness concerning critically important antibiotics, Fessler et al. (2011) emphasized that the usage of agents not listed as critical also holds the danger of spreading resistance against the latter agents. This is due to the fact that, in cases of selective pressure, several genes located on one mobile genetic element might be passed on together. Hence, it is essential to encourage a prudent use of all antibiotic agents and promote the assessment of alternative therapies (Ozawa et al., 2012). In this context, we propose that antibiotic drugs perceived as less critical should be given preference in the treatment of APM, even though our meta-analysis failed to generate evidence concerning comparative efficacy of antibiotic drugs.

Frequently, authors of systematic reviews or metaanalyses face the problem of missing studies with negative outcomes (Song et al., 2000), which is due to publication bias (i.e., the tendency to leave trials that fail to show superiority of the experimental treatment unpublished or to delay publication). On the other side, positive trials are more likely to be published more than once, which is not always readily identifiable by the reader. Publication bias can have an important effect on the outcomes of meta-analyses (Scifres et al., 2009); therefore, it is a main objective of meta-analyses to identify potential bias (Barker and Carter, 2005). Various approaches to quantify publication bias exist. The most widely applied method is the funnel plot, in which the effect size for individual trials is plotted against a measure of the precision of the treatment effect estimate, such as the size of the trial (Egger et al., 1997). In the present meta-analysis, the measure of precision was the standard error. When a funnel plot is used to study an unbiased sample of trials, the observed effect sizes should range symmetrically around the true effect size. As this will be most accurately estimated by the largest trials, a symmetrical, funnel-shaped plot should evolve (Barker and Carter, 2005). In the present funnel plot of the effect of a ceftiofur treatment on metritis prevalence following treatment, the included trials indeed range symmetrically around the true effect size; a funnel shape, however, could not be detected. In many cases, a missing funnel shape is due to a lack of small trials not providing much evidence of efficacy, and therefore not being published or retrieved (Barker and Carter, 2005). In the present investigation, however, a general shortage of studies with higher standard error regardless of the treatment outcome (positive or negative) existed. This suggests a publication bias toward smaller studies. Nevertheless, assessing the shape of a funnel plot is difficult when the number of trials included is small (Sterne et al., 2000). Due to the problem of limited number of trials, an investigation regarding publication bias for the other comparisons was omitted.

Besides promoting the performance of more highquality studies, our findings should also encourage reviewers in peer review processes and journal editors to more positively consider manuscripts for publication even though they do not provide novel information but merely confirm previous findings. Publication of reproduced findings lays the foundation for meta-analytic studies representing the highest evidence level possible (Bassler and Antes, 2000). This might be particularly important for critically perceived areas of food animal medicine. 


\section{CONCLUSIONS}

In conclusion, our meta-analysis disclosed a shortage of comparable studies and, hence, a need for more highquality studies. Furthermore, meta-analytic investigations revealed a positive effect of the most commonly used antibiotic drug, ceftiofur, for the treatment of bovine metritis. An advantage or disadvantage over other antibiotic agents, however, could not be investigated. Due to the limited number of comparable trials, metaanalytic comparison between the effect of antibiotic and nonantibiotic treatment options was not feasible. Hence, more research is warranted to study potentially promising nonantibiotic treatment approaches.

\section{REFERENCES}

Arlt, S., and W. Heuwieser. 2005. Evidence based veterinary medicine. Dtsch. Tierarztl. Wochenschr. 112:146-148.

Arlt, S. P. 2016. The staircase of evidence-A new metaphor displaying the core principles of evidence-based veterinary medicine. Vet. Evid. https://doi.org/10.18849/ve.v1i1.18.

Askie, L., and M. Offringa. 2015. Systematic reviews and meta-analysis. Semin. Fetal Neonatal Med. 20:403-409.

Azawi, O. I. 2008. Postpartum uterine infection in cattle. Anim. Reprod. Sci. 105:187-208.

Barker, F. G., 2nd, and B. S. Carter. 2005. Synthesizing medical evidence: Systematic reviews and metaanalyses. Neurosurg. Focus 19:E5.

Bassler, D., and G. Antes. 2000. Textbook of evidence-based medicine in clinic and practice [Lehrbuch evidenzbasierte medizin in klinik und praxis]. 1st ed. R. Kunz, G. Ollenschläger, H. Raspe, G. Jonitz, and F. W. Kolkmann, ed. Köln.

Beagley, J. C., K. J. Whitman, K. E. Baptiste, and J. Scherzer. 2010. Physiology and treatment of retained fetal membranes in cattle. J. Vet. Intern. Med. 24:261-268.

Bicalho, R. C., V. S. Machado, M. L. Bicalho, R. O. Gilbert, A. G. Teixeira, L. S. Caixeta, and R. V. Pereira. 2010. Molecular and epidemiological characterization of bovine intrauterine Escherichia coli. J. Dairy Sci. 93:5818-5830.

Borenstein, M., L. V. Hedges, J. P. T. Higgins, and H. R. Rothstein. 2009. Introduction to Meta-Analysis. John Wiley \& Sons, Wiltshire, UK.

Buchanan, R. A., and A. A. Wooldridge. 2011. Staying current by searching the veterinary literature. J. Vet. Med. Educ. 38:10-15.

Caldwell, D. M., A. E. Ades, and J. P. Higgins. 2005. Simultaneous comparison of multiple treatments: Combining direct and indirect evidence. BMJ 331:897-900.

Chaudari, C. F., V. S. Dabas, N. F. Chaudhari, and L. C. Modi. 2012. Bovine puerperal metritis and its managements-A report of two cases. Intas. Polivet. 13:34-35.

Chenault, J. R., J. F. McAllister, S. T. Chester Jr., K. J. Dame, F. M. Kausche, and E. J. Robb. 2004. Efficacy of ceftiofur hydrochloride sterile suspension administered parenterally for the treatment of acute postpartum metritis in dairy cows. J. Am. Vet. Med. Assoc. 224:1634-1639.

de Boer, M. W., S. J. LeBlanc, J. Dubuc, S. Meier, W. Heuwieser, S. Arlt, R. O. Gilbert, and S. McDougall. 2014. Invited review: Systematic review of diagnostic tests for reproductive-tract infection and inflammation in dairy cows. J. Dairy Sci. 97:3983-3999.

Drillich, M., O. Beetz, A. Pfutzner, M. Sabin, H. J. Sabin, P. Kutzer, H. Nattermann, and W. Heuwieser. 2001. Evaluation of a systemic antibiotic treatment of toxic puerperal metritis in dairy cows. J. Dairy Sci. 84:2010-2017.
Drillich, M., D. Voigt, D. Forderung, and W. Heuwieser. 2007. Treatment of acute puerperal metritis with flunixin meglumine in addition to antibiotic treatment. J. Dairy Sci. 90:3758-3763.

Duffield, T. F., A. R. Rabiee, and I. J. Lean. 2008. A meta-analysis of the impact of monensin in lactating dairy cattle. Part 1. Metabolic effects. J. Dairy Sci. 91:1334-1346.

Egger, M., G. Davey Smith, M. Schneider, and C. Minder. 1997. Bias in meta-analysis detected by a simple, graphical test. BMJ 315:629-634.

Federal Office of Consumer Protection and Food Safety. 2014. Germap 2012 - Bericht Über den Antibiotikaverbrauch und die Verbreitung von Antibiotikaresistenzen in der Human- und Veterinärmedizin in Deutschland. Antiinfectives Intelligence GmbH, Rheinbach, Germany.

Fessler, A. T., K. Kadlec, and S. Schwarz. 2011. Novel apramycin resistance gene apma in bovine and porcine methicillin-resistant Staphylococcus aureus st398 isolates. Antimicrob. Agents Chemother. 55:373-375.

Fishman, N. 2006. Antimicrobial stewardship. Am. J. Infect. Control 34(Suppl.):S55-S63. https://doi.org/10.1016/j.ajic.2006.05.237.

Földi, J., M. Kulcsar, A. Pecsi, B. Huyghe, C. de Sa, J. A. Lohuis, P. Cox, and G. Huszenicza. 2006. Bacterial complications of postpartum uterine involution in cattle. Anim. Reprod. Sci. 96:265-281.

Fourichon, C., H. Seegers, and X. Malher. 2000. Effect of disease on reproduction in the dairy cow: A meta-analysis. Theriogenology 53:1729-1759.

Freeman, T. D. 1955. Treatment of bovine and porcine metritis: Report of ten cases. Vet. Med. 50:307-308.

Giuliodori, M. J., R. P. Magnasco, D. Becu-Villalobos, I. M. LacauMengido, C. A. Risco, and R. L. de la Sota. 2013. Metritis in dairy cows: Risk factors and reproductive performance. J. Dairy Sci. 96:3621-3631

Glass, G. V. 1976. Primary, secondary, and meta-analysis of research Educ. Res. 10:3-8.

Glasziou, P., G. H. Guyatt, A. L. Dans, L. F. Dans, S. Straus, and D. L. Sackett. 1998. Applying the results of trials and systematic reviews to individual patients. ACP J. Club 129:A15-A16.

Goshen, T., and N. Y. Shpigel. 2006. Evaluation of intrauterine antibiotic treatment of clinical metritis and retained fetal membranes in dairy cows. Theriogenology 66:2210-2218.

Haimerl, P., and W. Heuwieser. 2014. Invited review: Antibiotic treatment of metritis in dairy cows: A systematic approach. J. Dairy Sci. 97:6649-6661.

Haimerl, P., W. Heuwieser, and S. Arlt. 2013. Therapy of bovine endometritis with prostaglandin f2alpha: A meta-analysis. J. Dairy Sci. 96:2973-2987.

Hedges, L. V., and I. Olkin. 1985. Statistical Methods for Meta-Analysis. Academic Press, New York, NY.

Higgins, J. P., S. G. Thompson, J. J. Deeks, and D. G. Altman. 2003 Measuring inconsistency in meta-analyses. BMJ 327:557-560.

Jeremejeva, J., T. Orro, A. Waldmann, and K. Kask. 2012. Treatment of dairy cows with PGF2alpha or NSAID, in combination with antibiotics, in cases of postpartum uterine inflammation. Acta Vet. Scand. 54:45.

Lam, R. W., and S. H. Kennedy. 2005. Using metaanalysis to evaluate evidence: Practical tips and traps. Can. J. Psychiatry 50:167-174.

Lean, I. J., M. C. Lucy, J. P. McNamara, B. J. Bradford, E. Block, J. M. Thomson, J. M. Morton, P. Celi, A. R. Rabiee, J. E. Santos, W. W. Thatcher, and S. J. LeBlanc. 2016. Invited review: Recommendations for reporting intervention studies on reproductive performance in dairy cattle: Improving design, analysis, and interpretation of research on reproduction. J. Dairy Sci. 99:1-17.

LeBlanc, S. J. 2008. Postpartum uterine disease and dairy herd reproductive performance: A review. Vet. J. 176:102-114.

LeBlanc, S. J., T. F. Duffield, K. E. Leslie, K. G. Bateman, G. P. Keefe, J. S. Walton, and W. H. Johnson. 2002. Defining and diagnosing postpartum clinical endometritis and its impact on reproductive performance in dairy cows. J. Dairy Sci. 85:2223-2236.

Lima, F. S., A. Vieira-Neto, G. S. Vasconcellos, R. D. Mingoti, E. Karakaya, E. Sole, R. S. Bisinotto, N. Martinez, C. A. Risco, K. 
N. Galvao, and J. E. Santos. 2014. Efficacy of ampicillin trihydrate or ceftiofur hydrochloride for treatment of metritis and subsequent fertility in dairy cows. J. Dairy Sci. 97:5401-5414.

Machado, V. S., M. L. Bicalho, E. B. Meira Junior., R. Rossi, B. L. Ribeiro, S. Lima, T. Santos, A. Kussler, C. Foditsch, E. K. Ganda, G. Oikonomou, S. H. Cheong, R. O. Gilbert, and R. C. Bicalho. 2014. Subcutaneous immunization with inactivated bacterial components and purified protein of Escherichia coli, Fusobacterium necrophorum and Trueperella pyogenes prevents puerperal metritis in Holstein dairy cows. PLoS One 9:e91734.

Machado, V. S., M. L. Bicalho, R. V. Pereira, L. S. Caixeta, J. H. Bittar, G. Oikonomou, R. O. Gilbert, and R. C. Bicalho. 2012. The effect of intrauterine administration of mannose or bacteriophage on uterine health and fertility of dairy cows with special focus on Escherichia coli and Arcanobacterium pyogenes. J. Dairy Sci. 95:3100-3109.

Mann, S., J. D. Siler, D. Jordan, and L. D. Warnick. 2011. Antimicrobial susceptibility of fecal Escherichia coli isolates in dairy cows following systemic treatment with ceftiofur or penicillin. Foodborne Pathog. Dis. 8:861-867.

McLaughlin, C. L., E. Stanisiewski, M. J. Lucas, C. P. Cornell, J. Watkins, L. Bryson, J. K. Tena, J. Hallberg, and J. R. Chenault 2012. Evaluation of two doses of ceftiofur crystalline free acid sterile suspension for treatment of metritis in lactating dairy cows. J. Dairy Sci. 95:4363-4371.

McLaughlin, C. L., E. P. Stanisiewski, C. A. Risco, J. E. Santos, G. E. Dahl, R. C. Chebel, C. LaGrow, C. Daugherty, L. Bryson, D. Weigel, J. Hallberg, and M. J. Lucas. 2013. Evaluation of ceftiofur crystalline free acid sterile suspension for control of metritis in high-risk lactating dairy cows. Theriogenology 79:725-734.

Miller, A. N., E. J. Williams, K. Sibley, S. Herath, E. A. Lane, J. Fishwick, D. M. Nash, A. N. Rycroft, H. Dobson, C. E. Bryant, and I. M. Sheldon. 2007. The effects of Arcanobacterium pyogenes on endometrial function in vitro, and on uterine and ovarian function in vivo. Theriogenology 68:972-980.

Morcos, M., A. H. Elyas, E. E. A. Safwat, and A. A. Amer. 1988 Bovine mastitis concomitant with post-partum metritis. Assiut Veterinary Medical Journal 38:46-54.

Nak, Y., S. B. Dagalp, C. Cetin, D. Nak, F. Alkan, E. Borum, and B. Tuna. 2011. Course and severity of postpartum metritis cases following antibiotic and PGF2alpha administration in postpartum metritis cows infected with bohv-4. Transbound. Emerg. Dis. 58:31-36.

Overton, M., and J. Fetrow. 2008. Economics of postpartum uterine health. Pages 39-43 in Proc. Dairy Cattle Reproduction Council Convention, Omaha, Nebraska. Dairy Cattle Reproduction Council, Champaign, IL.
Ozawa, T., Y. Kiku, M. Mizuno, S. Inumaru, S. Kushibiki, H. Shingu, T. Matsubara, H. Takahashi, and T. Hayashi. 2012. Effect of intramammary infusion of RBGM-CSF on SCC and expression of polymorphonuclear neutrophil adhesion molecules in subclinical mastitis cows. Vet. Res. Commun. 36:21-27.

Pechlivanoglou, P., F. Abegaz, M. J. Postma, and E. Wit. 2015. An alternative parameterization of Bayesian logistic hierarchical models for mixed treatment comparisons. Pharm. Stat. 14:322-331.

Reppert, E. J. 2015. Evidence for the use of ceftiofur for treatment of metritis in dairy cattle. Vet. Clin. North Am. Food Anim. Pract. 31:139-149. (vii.).

Sannmann, I., O. Burfeind, R. Voigtsberger, and W. Heuwieser. 2013. Comparison of two monitoring and treatment strategies for cows with acute puerperal metritis. Theriogenology 79:961-969.

Santos, T. M., R. O. Gilbert, and R. C. Bicalho. 2011. Metagenomic analysis of the uterine bacterial microbiota in healthy and metritic postpartum dairy cows. J. Dairy Sci. 94:291-302.

Scifres, C. M., J. D. Iams, M. Klebanoff, and G. A. Macones. 2009. Metaanalysis vs. Large clinical trials: Which should guide our management? Am. J. Obstet. Gynecol. 200:484.e1-484.e5.

Sheldon, I. M., J. Cronin, L. Goetze, G. Donofrio, and H. J. Schuberth. 2009. Defining postpartum uterine disease and the mechanisms of infection and immunity in the female reproductive tract in cattle. Biol. Reprod. 81:1025-1032.

Sheldon, I. M., G. S. Lewis, S. LeBlanc, and R. O. Gilbert. 2006 Defining postpartum uterine disease in cattle. Theriogenology 65:1516-1530.

Song, F., A. J. Eastwood, S. Gilbody, L. Duley, and A. J. Sutton. 2000 Publication and related biases. Health Technol. Assess. 4:1-115.

Steffan, J., M. Agric, S. Adriamanga, and M. Thibier. 1984. Treatment of metritis with antibiotics or prostaglandin $\mathrm{f} 2$ alpha and influence of ovarian cyclicity in dairy cows. Am. J. Vet. Res. 45:1090-1094.

Sterne, J. A. C., D. Gavaghan, and M. Egger. 2000. Publication and related bias in meta-analysis: Power of statistical tests and prevalence in the literature. J. Clin. Epidemiol. 53:1119-1129.

Stojkov, J., M. A. von Keyserlingk, J. N. Marchant-Forde, and D. M. Weary. 2015. Assessment of visceral pain associated with metritis in dairy cows. J. Dairy Sci. 98:5352-5361.

Tragesser, L. A., T. E. Wittum, J. A. Funk, P. L. Winokur, and P. J. Rajala-Schultz. 2006. Association between ceftiofur use and isolation of escherichia coli with reduced susceptibility to ceftriaxone from fecal samples of dairy cows. Am. J. Vet. Res. 67:1696-1700.

WHO. 2011. Critically important antimicrobials for human medicine. www.who.int/foodsafety/publications/antimicrobials-third/en/.

Yadav, D. K. 2014. Therapeutic management of RFM associated septic metritis - A report of 6 bovines. Intas Polivet 13:34-35. 